\title{
A Prospective, Randomized, Placebo-controlled Comparative Study of Amino Acid Supplementation in Lactation Insufficiency
}

\author{
Nagarathnamma $\mathrm{R}^{1}$, Pooja Bhushan ${ }^{2}$, Trayambak Dutta ${ }^{3}$, Ezhil Arasan $\mathrm{R}^{4}$
}

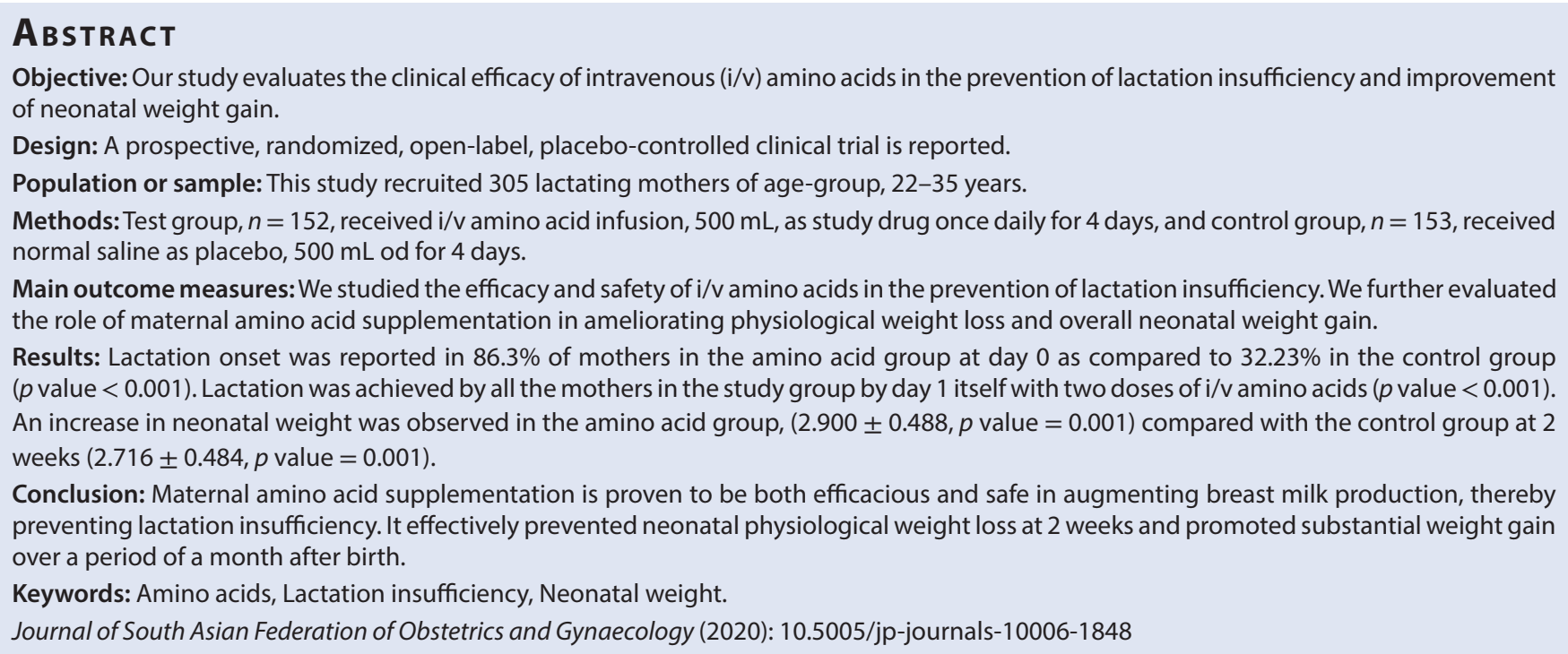

\section{INTRODUCTION}

Lactation insufficiency is a major issue of neonatal health. Poor breast milk production is the most frequent cause of lactation insufficiency. Inadequate milk production accounts for a substantial aggregate of lactation insufficiency cases worldwide. ${ }^{1}$ The World Health Organization recommends exclusive breastfeeding for the first 6 months of life starting 1 hour after delivery. ${ }^{2}$ Only $42 \%$ of newborns are put to the breast within the first hour of birth, globally and only two in five infants less than 6 months of age are exclusively breastfed. ${ }^{3}$ Breast milk substitutes increase the risk of mortality and morbidity among infants in developing countries and shorten birth intervals. Mothers delivering by cesarean section (C-section) are less likely to breastfeed or delay breastfeed initiation. ${ }^{4} \mathrm{C}$-sectiondelivered babies lack skin-to-skin contact immediately after birth with their mothers and the latter is more likely not to have attempted breastfeeding within the first 24 hours after delivery. Breastfeeding is associated with the infant's health benefits, such as improved intelligence as adults, lower cholesterol levels, stable blood pressure, fewer childhood illnesses, and lower prevalence of obesity. ${ }^{5}$ Preterm deliveries contribute to lactation insufficiency. ${ }^{6}$ In shortened pregnancies, mammary growth is incomplete and insufficiently primed for adequate lactogenesis. ${ }^{6} \mathrm{~A}$ low placental lactogen level along with poor placental function aggravates lactation insufficiency. ${ }^{6}$ Lactation is inhibited by fatigue, anxiety, and the stress of preterm delivery. Preterm mothers are incapable

\footnotetext{
1,2Department of Obstetrics and Gynaecology, Rajarajeswari Medical College and Hospital, Bengaluru, Karnataka, India

${ }^{3,4}$ Department of Medical and Scientific Affairs, Tablets India Limited, Chennai, Tamil Nadu, India

Corresponding Author: Trayambak Dutta, Department of Medical and Scientific Affairs, Tablets India Limited, Chennai, Tamil Nadu, India, Phone: +91 8939719805, e-mail: td@tabletsindia.com

How to cite this article: Nagarathnamma R, Pooja Bhushan, Dutta T, Ezhil Arasan R. A Prospective, Randomized, Placebo-controlled Comparative Study of Amino Acid Supplementation in Lactation Insufficiency. J South Asian Feder Obst Gynae 2020;12(6):408-414.

Source of support: Nil

Conflict of interest: None
}

of responding to stimulation of milk synthesis and expression, thereby leading to lactation insufficiency. ${ }^{7}$ Lack of breastfeeding in preterm infants is associated with a 2.4 -fold risk of necrotizing enterocolitis. Since the case fatality rate for necrotizing enterocolitis is $15 \%$, this difference in absolute risk is clinically significant. Poor maternal nutritional status especially before the onset of labor till the delivery of the newborn augments lactation insufficiency. A constant decrease in the levels of both maternal and fetal essential amino acid concentration is evident at full-term and pre-delivery. ${ }^{8}$ Animal studies have shown that amino acids can be considered as 
a valid therapeutic option to enhance milk production in lactation insufficiency with minimal side effects. Amino acids activate mammalian target of rapamycin (mTOR) signaling pathway in the mammary epithelial cells, thereby synthesizing milk proteins, i.e., caseins, $a$-lactalbumin, and $\beta$-lactoglobulins. This improves the quality of breast milk. Although animal studies are available, human clinical trials are lacking study efficacy and tolerability of intravenous (i/v) amino acids in the prevention of lactation insufficiency, and hence we did this study.

\section{Materials and Methods}

\section{Setting}

Rajarajeswari Medical College (RMC), Bengaluru, India, Department of Obstetrics, has 3,500 births per annum and is a tertiary referral center with level three neonatal intensive care facilities. The RMC Labor Ward is directly supervised by a senior registrar with 10-15 years of specialist training in obstetrics, 24 hours a day. In addition, there is one junior registrar (with 3 years of specialist training) and an intern trainee. Throughout the study period, there were at least 50 hours per week of direct consultant presence. Out of these periods, there was always indirect consultant supervision.

\section{Participants}

We examined the 305 nursing mothers of age-group 22-35 years.

\section{Case Identification and Data Validation}

For the 12-month period from October 2, 2018, to September 30, 2019, all pregnant women at term were screened by detailed medical history, vital signs, general, systemic examinations, and laboratory investigations. Mothers who delivered via normal vaginal delivery and $\mathrm{C}$-section were included while pregnancyinduced hypertension, renal failure, gestational diabetes mellitus, and elderly primigravida were excluded from the study. The final dataset was entered into an Excel spreadsheet and migrated into the department of statistical medicine for analysis. RMC ethical committee approved the study. This trial was registered at the Clinical Trials Registry of India (CTRI) vide no.-CTRI/2019/02/017650.

\section{Demographics and Other Confounders}

The following maternal demographic variables were collected; age at delivery, parity, mode of delivery, lactation onset, and urine output. Neonatal sleep quality, weight changes, and requirement of phototherapy were collected. The test group $(n=152)$ received i/v amino acid infusion, $500 \mathrm{~mL}$, od for 4 days and the control group ( $n=153$ ) received normal saline, i/v, $500 \mathrm{~mL}$, od for 4 days. Both placebo and study drug were administered 1 hour after delivery followed by a single dose every day till day 3 of birth. The study drug comprises isoleucine $5.6 \mathrm{mg}$, leucine $12.5 \mathrm{mg}$, lysine hydrochloride $11.0 \mathrm{mg}$, methionine $3.5 \mathrm{mg}$, phenylalanine $9.35 \mathrm{mg}$, threonine $6.5 \mathrm{mg}$, tryptophan $1.3 \mathrm{mg}$, valine $4.5 \mathrm{mg}$, histidine hydrochloride $8.11 \mathrm{mg}$, arginine hydrochloride $9.55 \mathrm{mg}$, alanine $6.2 \mathrm{mg}$, aspartic acid $3.8 \mathrm{mg}$, cysteine hydrochloride $1.45 \mathrm{mg}$, glutamic acid $6.5 \mathrm{mg}$, proline $3.3 \mathrm{mg}$, serine $2.2 \mathrm{mg}$, tyrosine $0.35 \mathrm{mg}$, glycine $10.7 \mathrm{mg}$, and xylitol $50.0 \mathrm{mg}$.

\section{Outcome Variables}

Maternal outcome variables collected were the efficacy of administration of $i / v$ amino acids in reducing the time of onset of lactation. Weight and sleep pattern were the neonatal outcomes measured.

\section{Statistical Analysis}

Pearson Chi-square test was used to evaluate parity, mode of delivery, lactation onset, urine output, baby sleep pattern, phototherapy requirement, and adverse drug reactions. $T$ test was employed to analyze intergroup comparison of gestational age and neonatal weight gain across and within the groups.

\section{Results}

This clinical trial included 305 patients with the control group, $n=152$, and the study group, $n=153$ patients, as per Figure 1 . The groups were evaluated with respect to parity, mode of delivery, lactation onset, urine output, sleep of the baby, and requirement of phototherapy. In terms of parity, $41.8 \%$ of the control group was primigravida and $45.1 \%$ were multigravida. In the study group, $45.1 \%$ were primigravida and $54.9 \%$ were multigravida. In the study group, $45.1 \%$ underwent lower segment cesarean section (LSCS), whereas $54.9 \%$ of the mothers delivered by vaginal delivery. In the control group, $41.2 \%$ underwent LSCS while $58.8 \%$ delivered by vaginal delivery (Table 1). At day 0 of birth, lactation onset was observed in $86.3 \%$ of the mothers in the amino acid group as compared to only $32.23 \%$ in the control group $(p<0.001)$ as depicted in Figure 2. Lactation was achieved by all the mothers in the study group by day 1 itself with two doses of $\mathrm{i} / \mathrm{v}$ amino acids $(p<0.001)$. The control group achieved $100 \%$ lactation only by day 3 . Physiological weight loss during the early weeks of birth was observed in the control group but not in the study group. Figure 3 demonstrates a statistically significant increase in weight as observed in the amino acid group, $(2.900 \pm 0.488$, $p=0.001)$ and a month after birth $(4.007 \pm 0.501, p<0.001)$

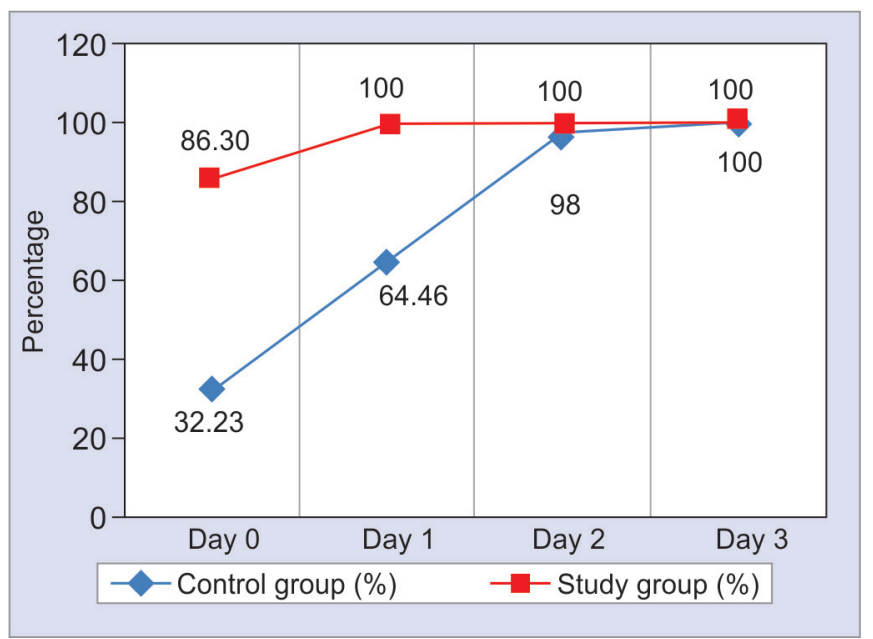

Fig. 1: CONSORT diagram

Table 1: Demographic data

\begin{tabular}{lllll}
\hline Parameters & Amino acids (\%) & Control (\%) & $p$ \\
\hline Mode of & C-section & 45.1 & 41.2 & \multirow{2}{*}{0.53} \\
delivery & Normal delivery & 54.9 & 58.8 & \\
\multirow{2}{*}{ Parity } & Primigravida & 45.1 & 41.8 & \multirow{2}{*}{0.56} \\
& Multigravida & 54.9 & 58.2 & \\
\hline
\end{tabular}


compared with the control group at 2 weeks $(2.716 \pm 0.484$ $p=0.001)$ and after a month after birth $(3.692 \pm 0.501, p<0.001)$. Weight gain was significantly higher in the treatment group at 2 weeks, $(2.900 \pm 0.488, p<0.001)$ and a month after birth $(4.007 \pm 0.501, p<0.001)$. Babies had better sleep in the amino acid group as compared to the control group ( $p<0.001$, Table 2 ). In the amino acid group, $90.8 \%$ of the babies had sound sleep compared to $69.9 \%$ in the control group. In terms of phototherapy, $83.7 \%$ of babies did not require phototherapy in the amino acid group compared to $68.7 \%$ in the control group ( $p=0.002$,Table 2 ). In the amino acid group, 2 patients reported itching at the site of injection and forearm while no adverse events were reported in the control group. Dropouts were registered due to excessive lactation in $24.8 \%$ of the cases in the amino acid group with the first dose compared to $0.7 \%$ cases in the control group $(p<0.001)$.

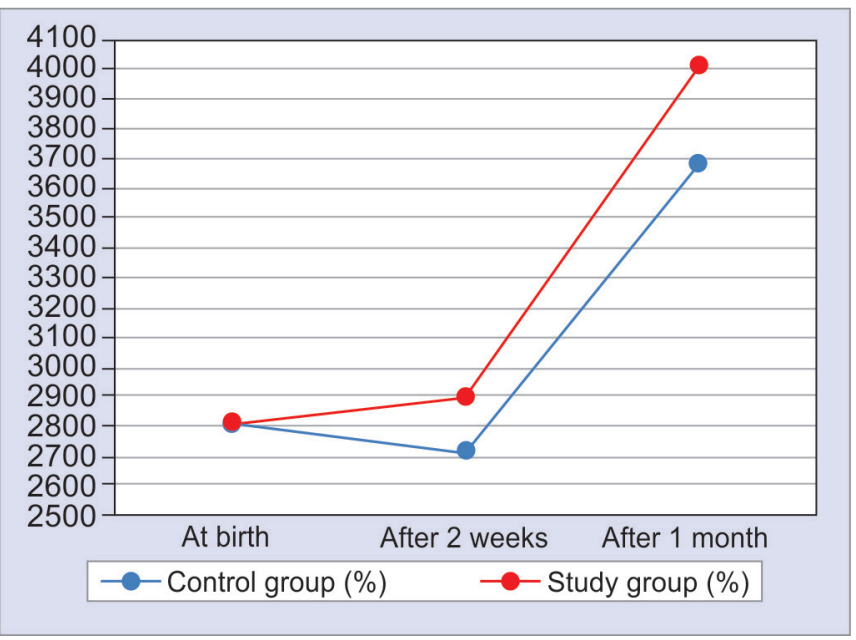

Fig. 2: Lactation onset

\section{Discussion}

To our knowledge, this is the first human clinical trial with 305 patients, which evaluates the clinical efficacy of $\mathrm{i} / \mathrm{v}$ amino acids in the prevention of lactation insufficiency and improvement of neonatal weight gain. Upon administering amino acids, $86.3 \%$ of the mothers undergoing $\mathrm{C}$-section achieved lactation on day 0 of birth compared to the control group ( $p<0.001)$. The lactation achieved by the study group was absolute by day 1 after delivery $(p<0.001)$. Weight gain achieved in the amino acid group was persistent compared to the control group. Weight gain was observed in the amino acid group $(2.900 \pm 0.488, p=0.001)$, and a month after birth $(4.007 \pm 0.501, p<0.001)$ as compared with the control group at 2 weeks $(2.716 \pm 0.484, p=0.001)$ and a month after birth $(3.692 \pm 0.501, p<0.001)$. Weight gain was reported within the amino acid group at 2 weeks $(2.900 \pm 0.488, p<0.001)$, and a month after birth $(4.007 \pm 0.501, p<0.001)$. Amino acid supplementation prevented the usual reduction in weight at 2 weeks after birth which is an important clinical outcome of our study. In an intergroup analysis, an increase of $200 \mathrm{gm}$ was noted between babies in the amino acid group as compared to the control group at 2 weeks (amino acid group: $2.9007 \pm 0.488$, control group: $2.7169 \pm 0.484$ $p<0.001)$. Lactation onset was observed in $86.3 \%$ of the mothers in the amino acid group at day 0 as compared to only $32.23 \%$ in the control group $(p<0.001)$.

In a randomized control trial in mammals, six lactating cows were randomly assigned to treatment groups with 21 days for each

Table 2: Phototherapy requirement and baby sleep

\begin{tabular}{llll}
\hline Parameters & Amino acid group (\%) & Control group (\%) & $p$ \\
\hline $\begin{array}{l}\text { Phototherapy } \\
\text { requirement }\end{array}$ & 16.3 & 31.4 & 0.002 \\
$\begin{array}{l}\text { Undisturbed } \\
\text { baby sleep }\end{array}$ & 90.8 & 69.9 & 0.000 \\
\hline
\end{tabular}

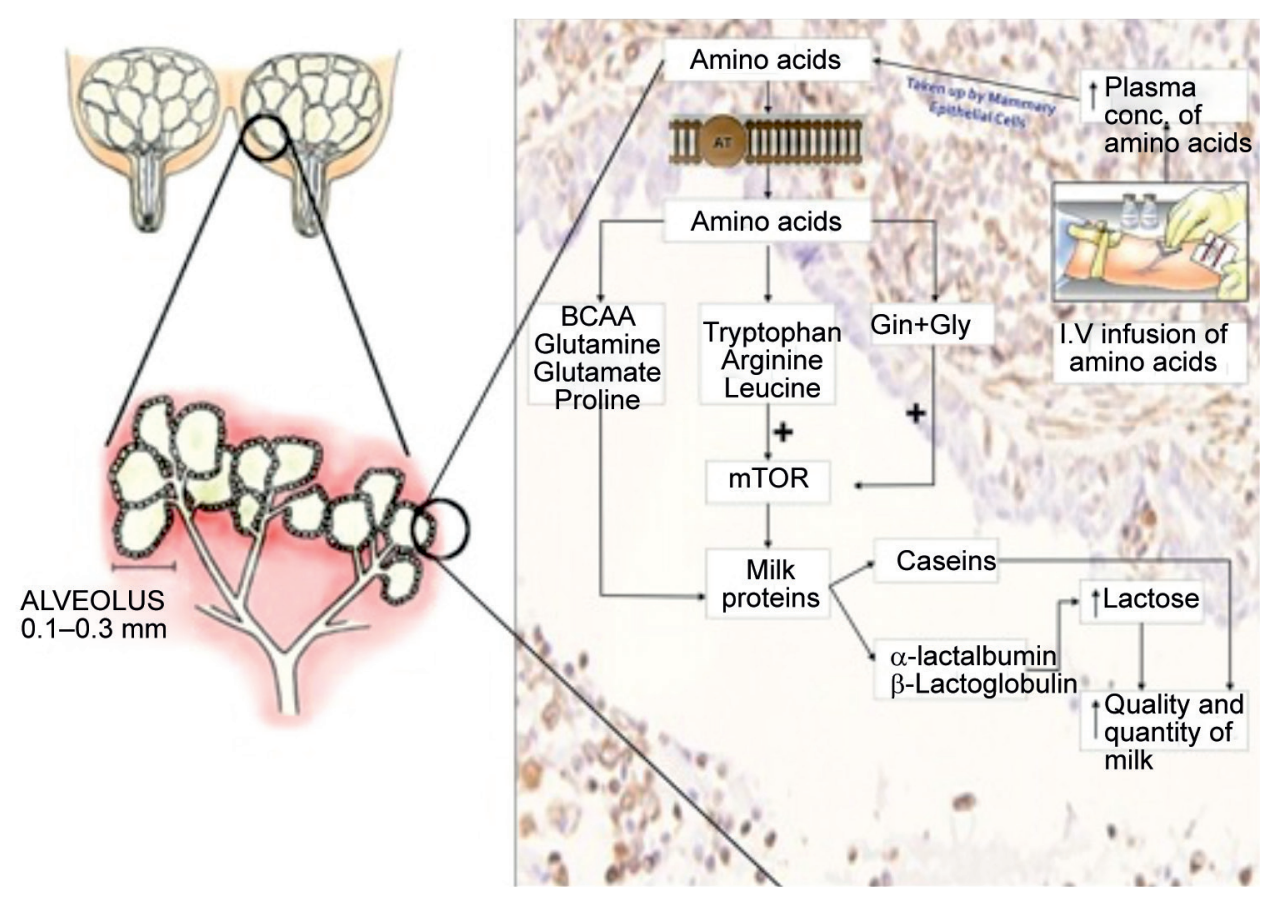

Fig. 3: Weight gain 
period. ${ }^{9}$ Control group received i/v saline. Study group received amino acid arginine (9.42 gm/L, L-arginine) and i/v saline. Milk production and composition, profiles of amino acids in blood, apparent absorption of nitrogen, and urea nitrogen in urine, milk, and blood were determined. The infusion of amino acid arginine led to greater expression of amino acid transporters (SLC7A2 and SLC7A8) and uptake of free amino acids in the mammary gland in comparison with the control group. It was accompanied by greater milk and milk protein yield and milk efficiency. A Canadian study reported women who delivered via emergency C-section were not able to breastfeed their baby at delivery or after discharge. ${ }^{4}$ Maternal and fetal stress response associated with complications during delivery, particularly related to C-sections, increases the difficulty and early cessation in breastfeeding during the early postpartum period. Lactogenesis is affected by an insult of abdominal surgery in both emergency and planned cesarean sections. ${ }^{4}$ The notion of an "emergency" invokes a prolonged maternal stress response affecting lactogenesis. A meta-analysis found an association between planned C-sections and the lack of breastfeeding initiation. ${ }^{10}$ Further, it was observed in another Mexican trial with 28 rural lactating women that amino acids can be channeled to tissue and milk protein synthesis or to catabolic pathways in the mammary gland. Glutamate-a metabolic product of the amino acid glutamine and other essential amino acids were found to be 40 -fold higher in milk with respect to plasma content. This suggests selective amino acid transport in the mammary tissue during lactation. It substantiates the fact that amino acids can be transferred to breast milk and hence to the neonate. Scattolin et al. reported improved short-term growth with neonatal amino acid intake. Neonatal head circumference and weight outcomes were better with amino acid supplementation as reported by Vlaardingerbroek et al. $(n=95)$, at 6 weeks after birth. ${ }^{11}$ From our study, we recommend early amino acid supplementation to the mothers starting day 0 of birth to maximize the transfer of the same to the infants which augments their weight gain. Lactation was achieved by all the mothers in the amino acid group by day 1 itself, ( $p<0.001)$. Dropouts were mainly because of excessive lactation. It was registered for $24.8 \%$ of the cases in the amino acid group with the first dose compared to $0.7 \%$ cases in the control group $(p<0.001)$.

Maternal amino acid supplementation leads to increased neonatal plasma amino acid concentration via breast milk. ${ }^{12}$ Essential amino acid transport is principal in neonatal hepatocellular development, thereby reducing the need for phototherapy in the amino acid group. ${ }^{13}$

\section{Mechanism of Amino Acids in Lactation}

Amino acids regulate metabolic pathways for whole-body homeostasis. They get catabolized in the lactating mammary tissue to provide amino groups for the biosynthesis of other amino acids, such as glutamine and glutamate, which are necessary for neonatal growth and digestive tract maturation of the baby. ${ }^{14}$ They regulate mammary gland metabolism. There are multiple mechanisms for amino acids to enhance breast milk production. Protein synthesis and subsequent enhanced milk production in the mammary epithelial cells via activation of the mTOR cell signaling pathway is depicted in Figure 4.

Amino acids enhance milk production in the following ways:

- I/v amino acid supplementation activates the mTOR pathway, thereby synthesizing the essential milk proteins, such as caseins, a-lactalbumin, and $\beta$-lactoglobulins.
Intravenous amino acid infusion

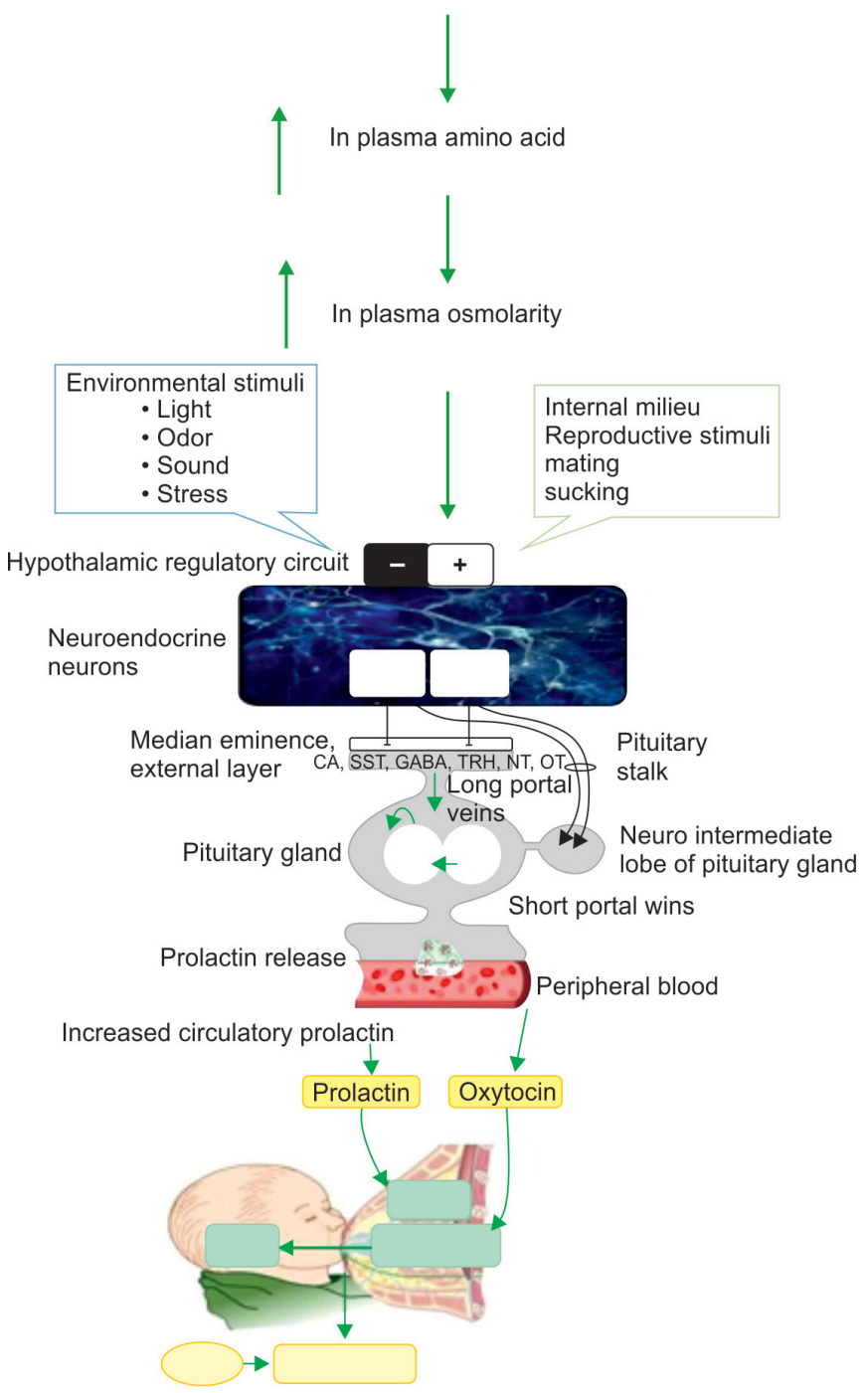

Fig. 4: Mechanism of action: amino acids in lactation

- Caseins are responsible for improving milk qualitatively and quantitatively. $a$-lactalbumins and $\beta$-lactoglobulins increase lactose content.

- Non-phosphorylated eukaryotic translation initiation factor 4E-binding protein 1 (4EBP1) inhibits protein synthesis by combining with the eukaryotic translation initiation factor $4 \mathrm{E}$ (elF4E). ${ }^{14}$

- Phosphorylation activates mTORC1 via 4EBP1 at multiple sites and subsequently causes the release of elF4E from 4EBP1-eIF4E complex.

- Once released, elF4E forms an active translation initiation complex. The translation initiation complex then binds the $40 \mathrm{~S}$ ribosomal subunit, generating the $43 \mathrm{~S}$ pre-initiation complex, thereby initiating translation of mRNA into protein. ${ }^{14}$

- The mTORC1 receptor is activated by amino acids, particularly arginine, leucine, glycine, tryptophan, and glutamine. ${ }^{15}$

- Leucine enhances overall protein synthesis by serving as a precursor for the synthesis of glutamine, aspartate, glutamate, and asparagine. ${ }^{14}$ 


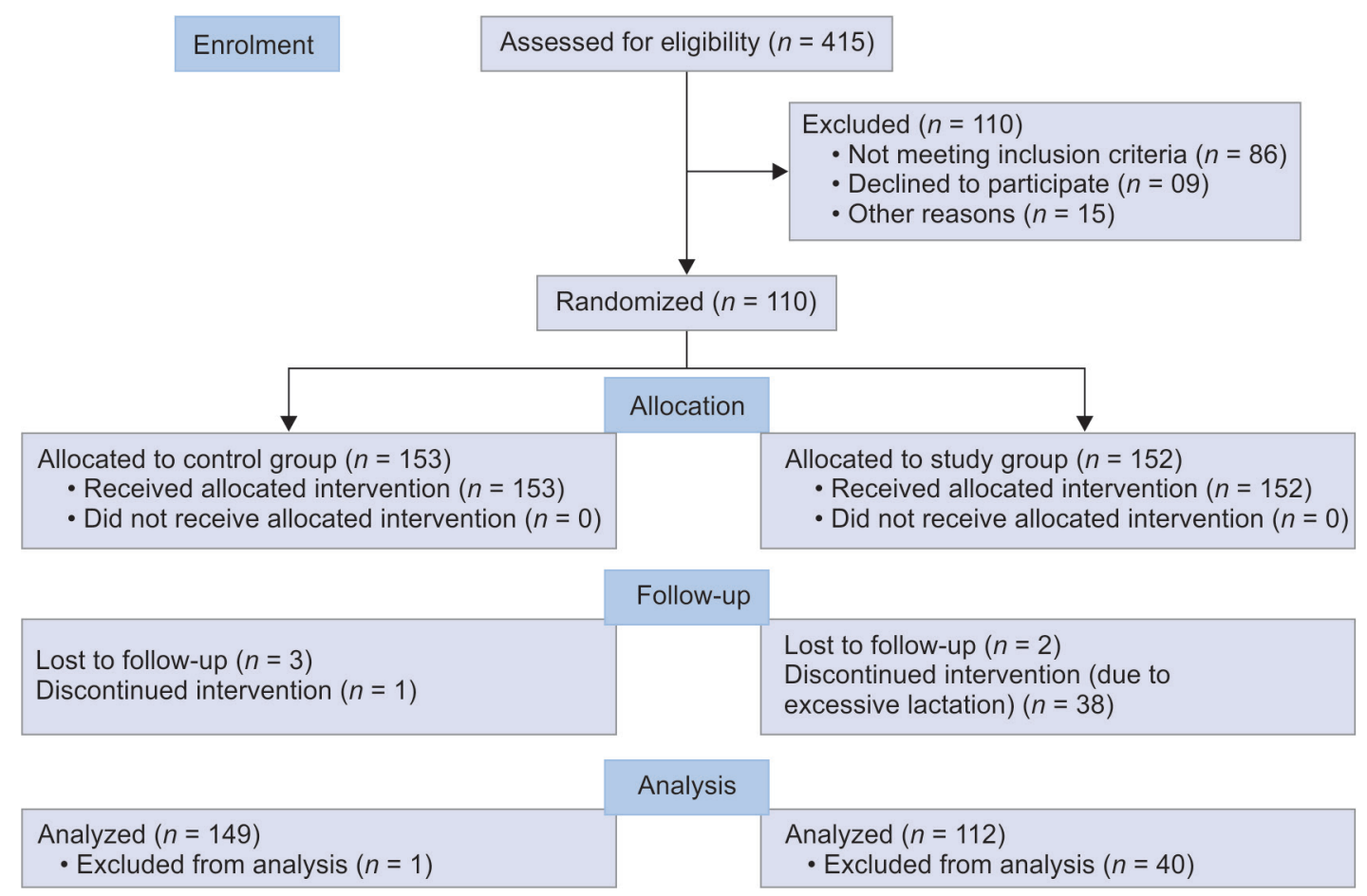

Fig. 5: Mechanism of action: amino acids in lactation

Constitutive proteins are actively synthesized by the cells of the mammary gland during lactation which accounts for $40-70 \%$ of total mammary protein synthesis. ${ }^{14}$ Mammals require large amounts of amino acids to support milk synthesis during lactation. The uptake of branched-chain amino acids by the mammary glands is much higher than their output in milk. Amino acids are catabolized extensively in lactating mammary tissue to provide amino groups for the biosynthesis of other amino acids, i.e., glutamine and glutamate which are necessary for neonatal digestive tract maturation and overall neonatal growth. The catabolism of amino acids by the mammary glandular epithelial cells is initiated by branched-chain amino acid transaminase in the presence of a-ketoglutarate to form branched-chain a-keto acids and glutamate. Thus, a concentration of branched-chain a-keto acids in milk is high and serves as energy substrates and differentiation of the small intestine of neonates. Substantial amounts of branched-chain a-keto acids are utilized by enterocytes of the small intestine and the splanchnic bed. They are responsible for the differentiation of the luminal epithelium of the mammary gland, thereby enhancing the milk-producing capacity of the same. Thus, amino acids are necessary for milk synthesis as well as for augmenting milk output. Figure 5 demonstrates the mechanism of $\mathrm{i} / \mathrm{v}$ amino acid administration in enhancing breast milk production. Upon i/v administration, there is an increase in plasma amino acid concentration, which augments plasma osmolarity. Along with other stimulants, such as light, sound, estradiol, glucocorticoids, and progesterone, increased plasma osmolarity activates the maternal hypothalamo-pituitaryadrenal (HPA) axis. ${ }^{16}$ The neuroendocrine neurons of the HPA axis release the prolactin regulatory factor which stimulates the neurointermediate lobe of the pituitary to release prolactin in the maternal circulation. ${ }^{17}$ In response to the suckling by the offspring, prolactin is responsible for the stimulation of milk synthesis in the mammary glands. Suckling by the baby activates ascending systems that decrease the release of dopamine, resulting in enhanced responsiveness to prolactin-releasing hormones, i.e., thyrotropin-releasing hormone. Premilk ejection, neurosecretory oxytocin population fires a synchronized burst of action potentials, resulting in the release of oxytocin from nerve terminals in the neurohypophysis. Oxytocin, synthesized in the magnocellular neurons of the supraoptic, hypothalamic, paraventricular, and accessory nuclei, is responsible for contracting the myoepithelial cells of the mammary gland to produce milk ejection. Neonatal growth, development, and health are sustained by human milk. Optimal health and growth of suckling neonates depends both quantitatively and qualitatively on milk production by their mothers. ${ }^{4}$ Milk synthesis of the lactating mammary gland is determined by the activity and the number of functional mammary epithelial cells, level of premature involution of the mammary gland, availability of precursors, stimulation of differentiation of various mammary epithelial cells, and concentration of lactogenic hormone. ${ }^{18}$ Lactogenic hormones, i.e., growth hormones, insulin, prolactin, and glucocorticoids are responsible for functional differentiation, proliferation, and involution of mammary epithelial cells. ${ }^{19}$ Translational and transcriptional regulation of gene expression of the mammary epithelial cells is modulated by lactogenic hormones. Amino acids regulate cell differentiation, cell cycle progression, cell apoptosis, and milk protein synthesis. ${ }^{20}$

Human mammary gland comprises epithelial cells, adipose tissue, and surrounding stroma. The epithelial cell in the lumen leads to synthesis and secretion of milk in the mammary gland. Milk upon synthesis is stored in the lactiferous tree duct. ${ }^{21}$ Upon suckling by the neonates, there is an oxytocin surge, which secretes milk. The synthesis of milk should be preceded by functional differentiation of the luminal epithelial cells. The quantity of functional mammary 
epithelial cells is important for the gland's milk synthetic capacity. ${ }^{21}$ Epithelial cells of the mammary gland undergo proliferation in both the pre- and post-partum periods for milk synthesis. Amino acids are the major components of milk proteins. They are nitrogenous precursors for the synthesis of glutamine, aspartate, alanine, and glutamate in the mammary gland. ${ }^{20}$ Amino acid transaminaseinitiated pathways lead to abundant peptide bound and free glutamate, aspartate, asparagines, and glutamine in milk. ${ }^{20}$ Amino acids modulate cell metabolism via synthesis of macromolecules (DNA and proteins), thereby promoting cell growth in the mammary epithelial cells. ${ }^{22}$ Constitutive proteins account for $40-70 \%$ of total mammary protein synthesis in lactation. ${ }^{14}$ Synthesis of constitutive proteins in the mammary tissue is proportional to the number of functional mammary epithelial cells. This physiological process is upregulated by amino acids.

Amino acids facilitate mammary gland maturation and differentiation by:

- Amino acids undergo protein synthesis by initiating mRNA translation by the mTOR pathway. ${ }^{23}$

- Amino acids stimulate phosphorylation of mTOR, thereby promoting translation initiation and polypeptide formation. ${ }^{20}$

- This results in growth, proliferation, and maturation of mammary epithelial cells.

This mechanism has important implications for the epigenetic regulation of lactogenesis. Amino acids, i.e., leucine and its metabolites, are secretagogues for insulin secretion by $\beta$-cells, partly through phosphorylation of mTOR downstream molecules p70S6K and 4EBP1, indicating the stimulatory role of amino acids in cell differentiation. ${ }^{20}$ In vitro and in vivo studies indicate that unlike the differentiating mammary epithelial cells, the undifferentiated and predifferentiated cells have low levels of mTOR phosphorylation. ${ }^{20}$ mTOR-mediated expression of the Id 2 protein (inhibitor of DNA-binding protein) is a prerequisite for cell differentiation in the mammary gland. ${ }^{24}$ Functional differentiation of mammary secretory cells and development of the mammary gland is executed by activation of the mTOR pathway. ${ }^{25}$ It explains the stimulating effect of amino acids on protein synthesis and milk secretion by mammary epithelial cells. There is a seven-fold increase in the mitochondrial inner membrane density during lactation. ${ }^{26}$ Thereby, there is an increase in the activity and the number of mitochondria in the lactating mammary tissue. However, adenosine triphosphate production is required to support high rates of milk production, yet endogenous free radicals generated from it concomitantly accumulate to negatively affect cell function. Amino acids increase the life span of mammary epithelial cells, thereby milk production via activating the mTOR signaling pathway. ${ }^{23} \mathrm{I} / \mathrm{v}$ amino acid supplementation can be used as a novel therapeutic approach for preventing lactation insufficiency and to improve neonatal weight gain outcomes.

\section{CONCLUSION}

Amino acid supplementation is proven to be both efficacious and safe in augmenting breast milk production, thereby preventing lactation insufficiency. Healthy, full-term, exclusively breastfed infants lose weight in the first days following birth. I/v amino acid supplementation effectively prevented this physiological weight loss and promoted substantial weight gain over a period of 1 month after birth. Amino acids should be considered for preventing lactation insufficiency, thereby promoting growth, weight gain, and development of the neonate.

\section{References}

1. Mehta A, Rathi AK, Kushwaha KP, et al. Relactation in lactation failure and low milk supply. Sudanese J Paediatr 2018 ;18(1):39-47. DOI: 10.24911/sjp.2018.1.6.

2. Pregnancy, childbirth, postpartum and newborn care: a guide for essential practice. 3rd ed. Geneva: World Health Organization; 2015.

3. UNICEF Data: Infant and young child feeding; July 2018.

4. Hobbs AJ, Mannion CA, McDonald SW, et al. The impact of caesarean section on breastfeeding initiation, duration and difficulties in the first four months postpartum. BMC Pregnancy Childbirth 2016;26;16:90. DOI: 10.1186/s12884-016-0876-1.

5. Hornell $A$, Lagstrom $H$, Lande $B$, et al. Breastfeeding, introduction of other foods and effects on health: a systematic literature review for the 5th Nordic Nutrition Recommendations. Food Nutr Res 2013;12:57. DOI: 10.3402/fnr.v57i0.20823.

6. Jones $E$, Spencer SA. Optimising the provision of human milk for preterm infants. Arch Dis Child Fetal Neonatal Ed 2007;92(4):F236F238. DOI: 10.1136/adc.2006.100941.

7. Asztalos EV. Supporting mothers of very preterm infants and breast milk production: a review of the role of galactogogues. Nutrients 2018;12;10(5):600. DOI: 10.3390/nu10050600.

8. Kerr GR. The free amino acids of serum during development of Macaca mulatta. II. During pregnancy and fetal life. Pediatr Res 1968;2(6):493-500. DOI: 10.1203/00006450-196811000-00007.

9. Ding $L$, Shen $Y$, Wang $Y$, et al. Jugular arginine supplementation increases lactation performance and nitrogen utilization efficiency in lactating dairy cows. J Animal Sci Biotechnol 2019;10:3. DOI: 10.1186/ s40104-018-0311-8.

10. Prior E, Santhakumaran S, Gale C, et al. Breastfeeding after cesarean delivery: a systematic review and meta-analysis of world literature. Am J Clin Nutr 2012;95(5):1113-1135. DOI: 10.3945/ajen.111.030254.

11. Vlaardingerbroek $H$, Vermeulen $M J$, Rook $D$, et al. Safety and efficacy of early parenteral lipid and high-dose amino acid administration to very low birth weight infants. J Pediatr 2013;163(3):638-644. DOI: 10.1016/j.jpeds.2013.03.059.

12. Zhang Z, Adelman AS, Rai D, et al. Amino acid profiles in term and preterm human milk through lactation: a systematic review. Nutrients 2013;5(12):4800-4821. DOI: 10.3390/nu5124800.

13. Rezaei R, Wu Z, Hou Y, et al. Amino acids and mammary gland development: nutritional implications for milk production and neonatal growth. J Anim Sci Biotechnol 2016;7:20. DOI: 10.1186/s40104-016-0078-8.

14. Taylor PM. Role of amino acid transporters in amino acid sensing. Am J Clin Nutr 2014;99(1):223S-230S. DOI: 10.3945/ajcn.113.070086.

15. Vedder H.Physiology of the hypothalamic-pituitary-adrenocortical axis. Neurolmmune Biol 2007;7:17-31. DOI: 10.1016/S1567-7443(07)00202-5.

16. Grattan DR.60 years of neuroendocrinology: the hypothalamo-prolactin axis. J Endocrinol 2015;226(2):T101-T122. DOI: 10.1530/JOE-15-0213.

17. Wall E, McFadden T. Regulation of mammary development as it relates to changes in milk production efficiency. September 26, 2012.

18. Macias $H$, Hinck L. Mammary gland development. Wiley Interdiscip Rev Dev Biol 2012;1(4):533-557. DOI: 10.1002/wdev.35.

19. Lei J, Feng $D$, Zhang $Y$, et al. Nutritional and regulatory role of branched-chain amino acids in lactation. Front Biosci (Landmark Ed) 2012;17:2725-2739. DOI: 10.2741/4082.

20. Pillay J, Davis TJ. Physiology, lactation. [Updated Apr 25, 2019]. In: StatPearls [Internet]. Treasure Island (FL): StatPearls Publishing; 2019.

21. Manjarin R, Bequette $B J, W u$ G, et al. Linking our understanding of mammary gland metabolism to amino acid nutrition. Amino Acids 2014;46(11):2447-2462. DOI: 10.1007/s00726-014-1818-8.

22. Zhou Y, Zhou Z, Peng J, et al. Methionine and valine activate the mammalian target of rapamycin complex 1 pathway through heterodimeric amino acid taste receptor (TAS1R1/TAS1R3) and intracellular $\mathrm{Ca}^{2+}$ in bovine mammary epithelial cells. J Dairy Sci 2018;101(12):11354-11363. DOI: 10.3168/jds.2018-14461. 
23. Jankiewicz M, Groner B, Desrivieres S. Mammalian target of rapamycin regulates the growth of mammary epithelial cells through the inhibitor of deoxyribonucleic acid binding Id 1 and their functional differentiation through Id2. Mol Endocrinol 2006;20(10):2369-2381. DOI: 10.1210/me.2006-0071.

24. Zhang $\mathrm{R}, \mathrm{MaH}$, Gao Y, etal. Th-POK regulates mammary gland lactation through mTOR-SREBP pathway. PLoS Genet 2018;8;14(2):e1007211. DOI: $10.1371 /$ journal.pgen.1007211.
25. Hadsell DL, Torres D, George J, et al. Changes in secretory cell turnover, and mitochondrial oxidative damage in the mouse mammary gland during a single prolonged lactation cycle suggest the possibility of accelerated cellular aging. Exp Gerontol 2006;41(3):271-281. DOI: 10.1016/j.exger.2005.12.005.

26. McManaman JL. Lipid transport in the lactating mammary gland. J Mammary Gland Biol Neoplasia 2014;19(1):35-42.DOI:10.1007/ s10911-014-9318-8 